\title{
Características endoscópicas e anatomopatológicas de uma série de 128 pólipos gástricos diagnosticados no Serviço de Endoscopia do Hospital Universitário Walter Cantídio
}

\section{Endoscopy and pathological features of a 128 gastric polyps serie diagnosed in the Endoscopy Unit of the Walter Cantidio University Hospital}

Suyanne Maria de Albuquerque Xerez Regadas ${ }^{1}$. Geraldo Cezário de Lázaro Filho². Sergio Henrique Brito Barbosa $^{3}$. José Telmo Valença ${ }^{4}$. Luciano Monteiro Franco ${ }^{5}$. Miguel Ângelo Nobre e Souza ${ }^{6}$. Marcellus Henrique Loiola Ponte de Souza?

1 Graduanda em Gastroenterologia com atuação em Endoscopia Digestiva, Universidade Federal do Ceará (UFC), Fortaleza, Ceará, Brasil. 2 Médico graduado em Endoscopia Digestiva, Universidade Federal do Ceará (UFC), Fortaleza, Ceará, Brasil. 3 Residente em Endoscopia Digestiva, Universidade Federal do Ceará (UFC), Fortaleza, Ceará, Brasil. 4 Patologista, Professor e Preceptor da Residência de Patologia, Universidade Federal do Ceará (UFC), Fortaleza, Ceará, Brasil. 5 Patologista e Servidor Técnico-administrativo, Departamento de Patologia, Universidade Federal do Ceará (UFC), Fortaleza, Ceará, Brasil. 6 Professor, doutor, Gastroenterologista e Endoscopista, Preceptor da Residência e Chefe do Serviço de Endoscopia Digestiva do Hospital Universitário Walter Cantídio (HUWC), Universidade Federal do Ceará (UFC), Fortaleza, Ceará, Brasil. 7 Professor, doutor, Gastroenterologista e Endoscopista, Preceptor da Residência e Chefe dos residentes do Serviço de Endoscopia Digestiva do Hospital Universitário Walter Cantídio (HUWC), Universidade Federal do Ceará (UFC), Fortaleza, Ceará, Brasil.

\section{RESUMO}

Objetivo: Avaliar a correlação entre o diagnóstico endoscópico e anatomopatológico dos pólipos gástricos e buscar possíveis associações. Metodologia: Estudo retrospectivo com análise de 128 pólipos em 121 pacientes , cujas informações foram colhidas a partir dos bancos de dados dos setores de endoscopia e patologia do HUWC-UFC, no período de maio de 2010 a maio de 2012. Resultados: $22 \%$ dos pacientes eram do sexo masculino e 78\% do feminino. Quanto às características endoscópicas, observamos mais comumente pólipos únicos, localizados no corpo e medindo menos que $1,0 \mathrm{~cm}, 60,9 \%$ dos pólipos eram hiperplásicos, $17,2 \%$ inflamatórios, 9,4\% de glândulas fúndicas e 4,6\% adenomas. A análise comparativa entre os adenomas e os demais pólipos definiu que $50 \%$ dos adenomas eram maiores que $1 \mathrm{~cm}$, enquanto que $88 \%$ dos não-adenomas eram menores que $1 \mathrm{~cm}(\mathrm{p}<0,05) .66,7 \%$ dos pólipos adenomatosos localizaram-se no antro, enquanto que os pólipos não-adenomatosos ocorreram em $31 \%$ dos casos (p =0,08). Conclusão: Houve predominância dos pólipos não-adenomatosos, principalmente do tipo hiperplásico. A associação da histologia de adenoma com pólipos maiores que $1,0 \mathrm{~cm}$ e localizados no antro foi encontrada.

Palavras-chave: Pólipos. Endoscopia. Biópsia. Neoplasias gástricas.

\section{ABSTRACT}

Purpose: To evaluate the correlation between the endoscopic and the pathologic diagnosis of gastric polyps, seeking any association. Methods: Retrospective study analyzing 128 polyps in 121 patients whose informations were collected from the databases of the Endoscopy and Pathology Units of the HUWC-UFC, from May 2010 to May 2012. Results: 22\% of patients were male and $78 \%$ female. Regarding the endoscopic characteristics, it was identified most commonly isolated polyps located in the stomach body and measuring less than $1.0 \mathrm{~cm}, 60,9 \%$ of the polyps were hyperplastic, $17,2 \%$ inflammatory, $9,4 \%$ fundic glands and $4,6 \%$ adenomas. The comparative analysis between adenomas and other polyps determined that 50\% of the adenomas were larger than $1 \mathrm{~cm}$, while $88 \%$ of non-adenomas were smaller than $1 \mathrm{~cm}(\mathrm{p}<0.05) .66 .7 \%$ of adenomatous polyps were located in the antrum, whereas non-adenomatous polyps were identified in $31 \%$ of the cases $(p=0.08)$. Conclusion: There was a prevalence of nonadenomatous polyps, especially the hyperplastic type. An association was found with adenoma histology when polyps were larger than $1.0 \mathrm{~cm}$ and located in the gastric antrum.

Keywords: Polyps. Endoscopy. Biopsy. Stomach neoplasms. 
Autor correspondente: Suyanne Maria de Albuquerque Xerez Regadas, Rua Carlota Pinheiro, 255 , 1701 B, Fortaleza, Ceará. CEP: 60810-019.

E-mail: suyannexerez@gmail.com

Conflito de interesses: Não há qualquer conflito de interesses por parte de qualquer um dos autores.

Recebido em: 07 Out 2015; Revisado em: 15 Out 2015; Aceito em: 19 Out 2015.

\section{INTRODUÇÃO}

Os pólipos gástricos são lesões elevadas da mucosa cuja incidência na literatura varia de $0,33-6,35 \% .^{1}$ Únicas ou múltiplas, podem ser encontradas em qualquer região do estômago. Em geral são menores que $1 \mathrm{~cm}$ e raramente causam sintomas. Quando grandes e próximos ao piloro podem ocasionar sintomas como obstrução intermitente e náuseas. $\mathrm{Na}$ presença de erosões podem evoluir com sangramento e cursar com melena ou anemia.

A maioria dos pólipos é encontrada incidentalmente durante exame de endoscopia digestiva alta (EDA) realizado por uma indicação não relacionada. Além de ser o método diagnóstico de escolha, tal procedimento permite avaliar as características macroscópicas dos pólipos, realizar biópsias e polipectomias terapêuticas. $^{2}$

As caracteristicas endoscópicas são definidas pela quantidade, tamanho, distribuição e aspecto das lesões. Comumente também utiliza-se a Classificação de Yamada. ${ }^{2}$

Já o estudo anatomopatológico dessas lesões epiteliais é essencial, pois identifica o tipo de lesão e seu respectivo potencial de malignidade .Os tipos de pólipos mais frequentes são os hiperplásicos (PH), inflamatórios (PIs), de glândulas fúndicas (PGFs) e adenomatosos (PAs). Entretanto existem outros tipos histológicos que fazem diagnóstico diferencial com essas lesões: tumores neuroendócrinos (carcinóides), heterotopia pancreática (pâncreas ectópico), xantelasmas, lipomas, leiomiomas, linfomas, etc. ${ }^{2}$

Neste presente estudo, buscou-se descrever as características demográficas, endoscópicas e anatomopatológicas dos pólipos gástricos encontrados no nosso serviço, comparando as possíveis associações.

\section{MATERIAIS E MÉTODOS}

Trata-se de um estudo retrospectivo a partir de informações colhidas de bancos de dados dos serviços de Endoscopia e Patologia do Hospital Universitário Walter Cantídio da Universidade Federal do Ceará (UFC), no período entre 26 de maio de 2010 e 08 de maio de 2012. Foram selecionados pacientes portadores de pólipos gástricos que realizaram endoscopia digestiva alta e polipectomia com pinça de biópsia ou alça diatérmica, dependendo do tamanho da lesão. A partir de um formulário padrão, analisou-se tanto aspectos demográficos, como endoscópicos e histopatológicos. A quantidade, localização e tamanho de cada lesão polipóide foi comparada. Além disso, patologistas experientes avaliaram e reavaliaram as mesmas amostras de tecido para confirmar a subclassificação anatomopatológica dessas lesões polipóides. Utilizou-se o programa estatístico Graph Pad Prisma e o teste de Fischer, considerando o $\mathrm{p}<0,05$ como estatisticamente significativo. Tal artigo faz parte de um projeto de pesquisa aprovado pelo comitê de ética.

\section{RESULTADOS}

\section{Características demográficas}

Cento e vinte e oito lesões polipóides gástricas foram identificadas em 121 pacientes. Houve 87 (72\%) mulheres e 34 (28\%) homens, com predomínio do sexo feminino $(2,5: 1)$ (Tabela 1).

\section{Características endoscópicas}

As características dos pólipos são mostrados na Tabela 1. A maioria dos pólipos, 73 (57\%), foi encontrado isoladamente, 2 a 4 pólipos ou múltiplos foram encontrados em 34 (26,5\%) e 21 (16,5\%), respectivamente. De 128 pólipos, 49 (38\%) foram localizados no corpo, 30 (23,5\%) no antro, 14 (11\%) no fundo, $13(10 \%)$ em cárdia, $12(9,5 \%)$ em antro e corpo e $10(8 \%)$ no fundo e corpo. $115(90 \%)$ pólipos mediram menos que $1,0 \mathrm{~cm}$, $11(8,4 \%)$ entre $1-3 \mathrm{~cm}$, somente $1(0,8 \%)$ mediu entre $3-5 \mathrm{~cm}$ e $1(0,8 \%)$ foi maior que $5 \mathrm{~cm}$.

\section{Características histopatológicas}

O subtipo histopatológico mais frequentes foi o hiperplásico, diagnosticado em $78(60,9 \%)$ das lesões, seguido dos pólipos inflamatórios com $22(17,2 \%)$, os de glândulas fúndicas com $12(9,4 \%)$ e os adenomas foram encontrados em $6(4,6 \%) .10$ $(7,9 \%)$ foram de outros subtipos (Tabela 1$)$.

\section{Análise comparativa entre os adenomas e os demais pólipos}

Realizou-se ainda uma análise comparativa entre os adenomas e os demais pólipos (Tabela 2) quanto ao tamanho, localização, quantidade e classificação de Yamada. O grupo 1 pertencia aos adenomas e o grupo 2 aos não-adenomas.

$88 \%$ dos pólipos não-adenomatosos e $50 \%$ dos adenomas eram menores que $1 \mathrm{~cm}$. 66,7\% do grupo 1 e $31 \%$ do grupo 2 foram detectados no antro. Em cerca de 50\% de ambos os grupos observou-se múltiplos pólipos. 9,8\% de pólipos Yamada III-IV foram vistos apenas no grupo dos pólipos nãoadenomatosos.

Em relação ao tamanho dos pólipos, observou-se que 50\% dos adenomas mediam mais de $1,0 \mathrm{~cm}$, diferentemente dos nãoadenomas, cuja maioria (88\%) era menor de $1,0 \mathrm{~cm}(\mathrm{p}<0,05)$. Os pólipos adenomatosos localizaram-se, predominantemente, no antro (66,7\%); já os pólipos não-adenomatosos ocorreram no antro em $31 \%$ dos casos; entretanto, não houve significância 
Tabela 1. Características dos pólipos gástricos.

\begin{tabular}{|c|c|c|}
\hline Características & & Número (\%) \\
\hline \multirow[t]{2}{*}{ Sexo } & Feminino & $72 \%(87 / 121)$ \\
\hline & Masculino & $28 \%(34 / 121)$ \\
\hline \multirow[t]{3}{*}{ Número } & 1 & $57 \%(73 / 128)$ \\
\hline & 2 a 4 & $26,5 \%(34 / 128)$ \\
\hline & Vários & $16,5 \%(21 / 128)$ \\
\hline \multirow[t]{6}{*}{ Localização } & Antro & $23,5 \%(30 / 128)$ \\
\hline & Corpo & $38 \%(49 / 128)$ \\
\hline & Fundo & $11 \%(14 / 128)$ \\
\hline & Fundo e corpo & $8 \%(10 / 128)$ \\
\hline & Antro e corpo & $9,5 \%(12 / 128)$ \\
\hline & Cárdia & $10 \%(13 / 128)$ \\
\hline \multirow[t]{4}{*}{ Tamanho } & $<1$ & $90 \%(115 / 128)$ \\
\hline & 1 a 3 & $8,4 \%(11 / 128)$ \\
\hline & 3 a 5 & $0,8 \%(1 / 128)$ \\
\hline & $>5$ & $0,8 \%(1 / 128)$ \\
\hline \multirow[t]{5}{*}{ Subtipo histológico } & Hiperplásico & $60,9 \%(78 / 128)$ \\
\hline & Inflamatório & $17,2 \%(22 / 128)$ \\
\hline & Glândulas fúndicas & $9,4 \%(12 / 128)$ \\
\hline & Adenomas & $4,6 \%(6 / 128)$ \\
\hline & Outros & $7,9 \%(10 / 128)$ \\
\hline
\end{tabular}

Tabela 2. Comparação das características dos Pólipos não-adenomatosos com os Pólipos adenomatosos.

\begin{tabular}{|c|c|c|c|}
\hline & Adenoma $(\mathrm{N}=6)$ & Não-adenoma $(\mathrm{N}=122)$ & $\mathrm{p}$ \\
\hline Tamanho $(<1 \mathrm{~cm})$ & $50 \%$ & $88 \%$ & $\mathrm{p}<0,05$ \\
\hline Localização (Antro) & $66,70 \%$ & $31 \%$ & $\mathrm{p}=0,08$ \\
\hline Múltiplos & $50 \%$ & $44 \%$ & $\mathrm{p}>0,05$ \\
\hline Yamada III-IV & 0 & $9,80 \%$ & $\mathrm{p}>0,05$ \\
\hline
\end{tabular}


estatística $(p=0,08)$. Em relação ao número de pólipos e à classificação de Yamada, não observou-se diferença estatística entre os dois grupos $(\mathrm{p}>0,05)$.

\section{DISCUSSÃO}

Os dados da literatura sobre pólipos gástricos mostram amplas variações tanto nos achados endoscópicos como nas prevalências dos diferentes subtipos histológicos. Acreditase que tamanha discrepância ocorra devido aos diferentes modos de estudo e pupulações, influenciadas ainda pelas características demográficas, genéticas e/ou socioeconômicas. ${ }^{2}$

No presente estudo, a análise endoscópica inicial detectou 128 pólipos em 121 pacientes, sendo a maioria mulheres (72\%). 73 lesões (57\%) foram encontradas isoladamente, coincidindo com as demais séries. Quanto à distribuição, observamos dados semelhantes na literatura, com variações de $21-24 \%$ em fundo, $38-40 \%$ em corpo e $35-40 \%$ em antro. ${ }^{2}$ Nossos achados foram predominantes em corpo e antro $(61,5 \%)$. Com relação ao tamanho, somente $10,2 \%$ dos pólipos analisados eram maiores que $1 \mathrm{~cm}$.

Com base na análise anatomopatológica, as prevalências encontradas na literatura variaram de 7,4-77\% para os pólipos de glândulas fúndicas, de 17-71,5\% para os hiperplásicos e de 0,69-23\% para os adenomas. ${ }^{2}$ Em nossa amostra, observou-se $60,9 \%$ tipo hiperplásico, 17,2\% inflamatórios e $9,4 \%$ de glândulas fúndicas. Os adenomas e outros tipos de lesões foram detectados em 4,6\% e 7,9\%, respectivamente. O conhecimento destes subtipos histológicos é essencial para definir conduta diante do risco de malignidade.

Pólipos hiperplásicos ou inflamatórios raramente acometem mucosa gástrica normal, sendo comumente associados à gastrite crônica ou atrófica e anemia perniciosa. Esses correspondem a $75 \%$ dos pólipos gástricos em regiões onde a infecção pelo H. pylori é comum. Estas associações aumentam o risco de adenocarcinomas sincrônicos ou metacrônicos. ${ }^{3}$ $\mathrm{O}$ risco de malignidade é maior para pólipos maiores que 1 $\mathrm{cm}$, mas o câncer invasivo é raro. ${ }^{4}$ Todos os pacientes com H. pylori devem ser submetidos a terapia de erradicação. ${ }^{5}$ Por tratar-se de um estudo retrospectivo, em alguns dos pacientes não foi possível avaliar a associação com o H .pylori.

Já os pólipos de glândulas fúndicas são frequentes nos países ocidentais, onde a taxa de infecção pelo H.pylori é menor e o uso crônico de IBP (Inibidor de bomba de protons) é comum. A terapia a longo prazo com IBP ( $\geq 5$ anos) foi associada a um risco quatro vezes maior para pólipos de glândulas fúndicas, enquanto a terapia de curto prazo $(<1$ ano) não teve a mesma relação. ${ }^{6}$ A maioria dessas lesões é do tipo esporádica, contudo estas podem vir associadas às síndromes de polipose e Zollinger-Ellison. ${ }^{7,8}$ Mutações no gene APC foram detectadas em mais de $70 \%$ dos pólipos no contexto de síndromes de polipose sem displasia. Já nos casos de pólipos esporádicos, a prevalência destas mutações foi inferior a $10 \%{ }^{9}$

Os adenomas constituem o tipo mais comum de pólipo gástrico neoplásico. ${ }^{10}$ Localizam-se mais frequentemente na incisura angular e antro. Cerca de $30 \%$ podem ser precursores do adenocarcinoma gástrico. Segundo estudo, pacientes com displasia de alto grau têm um maior risco de progressão para adenocarcinoma invasivo, quando comparados aos pacientes com displasia de baixo grau. ${ }^{11}$

Para uma análise adequada dos pólipos gástricos devemos avaliar a histologia do pólipo e da mucosa circundante. Lesões polipóides gástricas únicas e pequenas, deve-se proceder a polipectomia ou a coleta de amostras do pólipo. Nos casos de pólipos neoplásicos conhecidos e pólipos com diâmetro maior que $1 \mathrm{~cm}$, a polipectomia deve ser realizada, visto que amostras de biópsia isoladas não nos permitem excluir a presença de focos de displasia de alto grau ou câncer gástrico precoce. ${ }^{12}$ Em pacientes com múltiplos pólipos, o maior pólipo deve ser ressecado e biópsias representativas dos pólipos restantes devem ser obtidas. ${ }^{13}$

Condutas complementares podem ser definidas a partir dos resultados da histologia. ${ }^{2}$ Pólipos hiperplásicos sem displasia ou carcinoma, o acompanhamento deve basear-se no risco de câncer devido a gastrite crônica atrófica associada e nos fatores de risco concomitantes para o câncer gástrico. Tendo em vista que os pólipos de glândulas fúndicas são múltiplos na maioria dos casos, biópsias de um ou mais pólipos representativos são suficientes para a avaliação histopatológica.

Tendo em vista o risco aumentado para câncer gástrico, todos os adenomas gástricos devem ser ressecados. Em geral, a ressecção pode ser realizada por via endoscópica. No entanto, a cirurgia pode ser necessária para lesões que contenham carcinoma invasivo ou em pacientes com múltiplos adenomas. Considerando a associação entre displasia gástrica e carcinoma gástrico sincrônico, todo o restante do estômago deve ser cuidadosamente examinado. ${ }^{13}$

Nossa serie de casos possibilitou realizar uma análise comparativa entre os pólipos não-adenomatosos e os adenomatosos. Os pólipos localizados em antro e medindo acima de $1 \mathrm{~cm}$, apresentaram associação com os pólipos adenomatosos, demonstrando uma importante correlação entre os achados endoscópicos e histopatológicos. Entretanto, sabese que ainda são necessários estudos com casuística maior, prospectivos, e de preferência multicêntricos, para definir um verdadeiro protocolo de acompanhamento dos pacientes portadores de pólipos gástricos. 


\section{REFERÊNCIAS}

1. Dirschmid K, Platz-Baudin C, Stolte M. Why is the hyperplastic polyp a marker for the precancerous condition of the gastric mucosa? Virchows Arch. 2006;448(1):80-4.

2. Sugai BM, Yamazaki K. Pólipos gástricos. In: Averbach M, Safatle-Ribeiro AV, Junior AP, Cappellanes CA, Ejima FH, Fang HL, editores. Endoscopia digestiva: diagnóstico e tratamento. Rio de Janeiro: Revinter; 2013. p. 317-20.

3. Antonioli, DA. Precursors of gastric carcinoma: a critical review with a brief description of early (curable) gastric cancer. Hum Pathol. 1994;25(10):994-1005.

4. Ohkusa T, Takashimizu I, Fujiki K, Suzuki S, Shimoi K, Horiuchi $\mathrm{T}$, et al. Disappearance of hyperplastic polyps in the stomach after eradication of Helicobacter pylori. A randomized, clinical trial. Ann Intern Med. 1998;129(9):712-5.

5. Jalving M, Koornstra JJ, Wesseling J, Boezen HM, De Jong S, Kleibeuker JH. Increased risk of fundic gland polyps during longterm proton pump inhibitor therapy. Aliment Pharmacol Ther. 2006;24(9):1341-8.

6. Lynch HT, Smyrk T, McGinn T, Lanspa S, Cavalieri J, Lynch $\mathrm{J}$, et al. Attenuated familial adenomatous polyposis (AFAP). A phenotypically and genotypically distinctive variant of FAP. Cancer. 1995;76(12):2427-33.

7. Modlin IM, Gilligan CJ, Lawton GP, Tang LH, West AB, Darr U. Gastric carcinoids. The Yale Experience. Arch Surg. 1995;130(3):250-5.
8. Abraham SC, Nobukawa B, Giardiello FM, Hamilton SR, Wu TT. Fundic gland polyps in familial adenomatous polyposis: neoplasms with frequent somatic adenomatous polyposis coli gene alterations. Am J Pathol. 2000;157(3):747-54.

9. Rugge M, Nitti D, Farinati F, di Mario F, Genta RM. Noninvasive neoplasia of the stomach. Eur J Gastroenterol Hepatol. 2005;17(11):1191-6.

10. De Vries AC, Van Grieken NC, Looman CW, Casparie MK, De Vries E, Meijer GA, et al. Gastric cancer risk in patients with premalignant gastric lesions: a nationwide cohort study in the Netherlands. Gastroenterology. 2008;134(4):945-52.

11 Muehldorfer SM, Stolte M, Martus P, Hahn EG, Ell C, et al. Diagnostic accuracy of forceps biopsy versus polypectomy for gastric polyps: a prospective multicentre study. Gut. 2002;50(4):465-70.

12 ASGE Standards of Practice Committee; Sharaf RN, Shergill AK, Odze RD, Krinsky ML, Fukami N, et al. Endoscopic mucosal tissue sampling. Gastrointest Endosc. 2013;78(2):216-24.

13. Rugge M, Meggio A, Pennelli G, Piscioli F, Giacomelli L, De Pretis G, et al. Gastritis staging in clinical practice: the OLGA staging system. Gut. 2007;56(5):631-6.

\section{Como citar:}

Regadas SM, Lázaro GC Filho, Barbosa SH, Valença JT, Franco LM, Souza MA, Souza MH. Características endoscópicas e anatomopatológicas de uma série de 128 pólipos gástricos diagnosticados no Serviço de Endoscopia do Hospital Universitário Walter Cantídio. Rev Med UFC. 2015 jul-dez;55(2):23-27. 